\title{
Laparoscopic versus open inguinal spermatic vessel ligation in infertile men with varicocele.
}

\author{
Shuang Liu, Chuanyi Hu*, Ning Jiang, Guozeng Wang \\ Department of Urology, Shanghai Pudong New Area Gongli Hospital, Shanghai, PR China
}

\begin{abstract}
Objective: Varicocele in infertile men is effectively treated by either Laparoscopic or Open Inguinal Spermatic Vessel Ligation (L-SVL and OI-SVL, respectively), but comparative information is limited. This retrospective study compared the outcomes and complications of L-SVL and OI-SVL.

Methods: Infertile men with varicocele were treated with L-SVL $(n=140 ; 2001-2006)$ or OI-SVL $(n=120$; 2007-2012). Each patient had infertility $>1$ y; abnormal semen parameters; no other infertility-related disease; no obvious causes of infertility in the partner; and basal eco-color Doppler ultrasound showed continuous reflux in the spermatic vessel. Evaluations were conducted regarding rates of recurrence, intraoperative and postoperative complications, operative time, hospitalization, cost, and semen parameters 6 months after surgery.

Results: The demographics and preoperative (baseline) clinical features of the 2 groups were similar. Fewer men in the L-SVL group $(\mathbf{0 . 8 3} \%)$ had recurrence of the varicocele after surgery compared with the OI-SVL group $(2.86 \%, P<0.01)$, and men given $\mathrm{L}-\mathrm{SVL}$ experienced significantly less time in surgery, recovery, and hospital stay. The groups were similar regarding rates of wound complications, damage to the genitofemoral nerve, testicular atrophy, epididymo-orchitis, and hydrocele, and procedural cost. Compared with the baseline, both groups showed significant postoperative improvements in sperm count, motility, and morphology $(\mathbf{P}<0.01)$, while the postoperative findings of the 2 groups were similar. Conclusion: Both L-SVL and OI-SVL were effective in correcting varicocele in men, but L-SVL was associated with a lower varicocele recurrence rate, shorter hospitalization, better security, and healing rate.
\end{abstract}

Keywords: Varicocele, Laparoscopic spermatic vessel ligation, Male infertility.

Accepted on January 23, 2018

\section{Introduction}

Varicocele is a dilatation of the testicular veins due to retrograde blood flow caused by valve incompetency. In $35 \%$ of men with primary infertility and $80 \%$ of men with secondary infertility, varicocele is the most commonly correctable cause $[1,2]$. The prevalence of varicocele is reportedly as high as $20-24 \%$ in the adult male population, with a higher prevalence on the left side [3]. Varicocele causes infertility in a number of ways, by reducing sperm count, motility, and testicular hormone production. Morphological changes have also been reported in the sperm of men with varicocele. These factors can therefore be used as markers for the early stages of varicocele. Otherwise, varicocele is usually diagnosed by physical examination of the scrotum and the spermatic cord, using the Valsalva maneuver with the patient standing.

There is no definitive explanation for the association between infertility and varicocele. However, when an infertile man is found to possess a varicocele the general consensus is that spermatic vessel ligation should be considered as the first therapeutic step, regardless the severity of varicocele.
Furthermore, some young patients with varicocele, and especially those in whom varicocele was found by chance, become deeply concerned over the potential influence of this condition upon their subsequent fertility.

For the clinical management of varicocele via varicocelectomy, approaches include Microscopic Subinguinal Varicocelectomy (MSV), Laparoscopic Spermatic Vessel Ligation (L-SVL), and Open Inguinal Spermatic Vessel Ligation (OI-SVL). Although MSV is the first-line treatment for VC in infertile men [4], open surgery and laparoscopic surgery are still the most popular method in primary hospitals which lack appropriate microscopes. OI-SVL is currently the most common approach for the treatment of varicocele, there is evidence that L-SVL is minimally invasive, rapid, safe, and effective $[5,6]$. The present retrospective study evaluated L-SVL relative to OI-SVL with regard to outcomes, complications. 


\section{Methods}

\section{Patient data}

The protocol for the diagnosis and treatment of varicocele in our Department was established in 2001. Patients were considered for surgery when they exhibited a symptomatic varicocele accompanied by an alteration in seminal fluid, thus suggesting impairment of fertility. Infertility was defined, according to the World Health Organization, as the inability of a sexually active couple, who are not using any contraceptive, to achieve pregnancy within one year. All the patients in this study were adults (aged $>18 \mathrm{y}$ ). Prior to the operation, at least 2 semen samples were obtained by masturbation after $3 \mathrm{~d}$ of abstinence. The diagnosis of varicocele was based upon physical examination and Doppler ultrasonography. The grade of varicocele severity was determined through physical examination in a standing position, in accordance with published criteria [7].

From 1 January 2001 to 31 December 2006, 140 patients underwent OI-SVL surgery whereas from 1 January 2007 to 31 December 2012, 120 patients underwent L-SVL surgery (Table 1). The L-SVL and OI-SVL groups were similar in preoperative (baseline) age; varicocele side, number, and grade; and indications for varicocelectomy.

\section{Surgical procedures}

All patients were admitted to our unit the day before surgery. Regardless of the technique, all patients underwent surgery under general anesthesia. In both groups, the bowel was prepared with a simple enema. Antibiotics were not administered to either of the two groups.

OI-SVL surgery was performed based on the Palomo technique [8]. First, the skin was incised over the inguinal ring. The length of the incision was less than $3 \mathrm{~cm}$ and the veins were ligated at the internal inguinal ring.

L-SVL surgery was performed by applying three ports (a 10 $\mathrm{mm}$ umbilical port for the lens, and two $5 \mathrm{~mm}$ ports for surgical tools) after the patient received general endotracheal anesthesia and was positioned supine in a modest Trendelenburg posture. Locations of the trocars, and the surgical procedures deployed, were similar to those of the conventional transperitoneal laparoscopic technique. First, a trocar was inserted using a modified open access technique, with a $10 \mathrm{~mm}$ transverse umbilical incision; the peritoneum was opened under direct vision. A pneumoperitoneum was created (using up to $12-15 \mathrm{mmHg}$ carbon dioxide). Two working trocar ports were placed under direct vision at the lateral border of each abdominal rectus muscle, at a level $1 \mathrm{~cm}$ than the umbilicus.

After dissecting the adhesion between the intestine/mesentery and the varicoceles, and after identifying the spermatic vessels (eventually pulling down the testis and stretching the vessels), a retroperitoneal incision was made in the lateral aspect from a point $3 \mathrm{~cm}$ superior to the internal inguinal ring along the testicular vessels. The testicular vessels were exposed. With blunt dissection, the vessels were carefully freed from underneath the peritoneum; they were then grasped, put on traction, clipped, and divided.

After the procedures were completed, the intraperitoneal pressure was reduced to $5 \mathrm{mmHg}$ to check for any subtle venous oozing, and adequate hemostasis was obtained. The trocars were subsequently removed with a suturing fascia for the $10 \mathrm{~mm}$ umbilical port wounds, and a simple skin closure for the $5 \mathrm{~mm}$ wounds.

\section{Follow-up data}

Treatment outcomes were assessed 6 months after surgery by physical examination, Doppler ultrasonography, and semen analysis in all patients.

\section{Statistical analysis}

Student's t-test was used for continuous variables, and the chisquare test for categorical variances. $\mathrm{P}<0.05$ was considered statistically significant. Data were analysed using an SAS 12.1 software package (SAS Institute, Cary, NC, USA).

\section{Results}

Operative procedures were smooth in all 260 patients. No major intraoperative complications occurred in either group (Table 2). The recurrence rate of varicocele was significantly lower in the L-SVL group (0.83\%) compared with the OI-SVL group $(2.86 \%, \mathrm{P}<0.01)$ at the 6-month follow-up. The operative time, recovery time, and duration of hospitalization were significantly shorter in the L-SVL group compared with the OI-SVL group $(\mathrm{P}<0.05$, each). The mean duration of hospitalization was 4.3 and $7.5 \mathrm{~d}$ in the L-SVL and OI-SVL groups, respectively. On average, the patients treated with LSVL recovered within $1.8 \mathrm{~d}$, whereas the patients treated with OI-SVL required $3.2 \mathrm{~d}$. There was no statistical difference between the groups in terms of the cost of each technique.

There were no significant differences between the groups regarding wound complications, damage to the genitofemoral nerve, testicular atrophy, epididymo-orchitis, or hydrocele. None of the patients reported testicular pain after laparoscopic ligation, and no reduction in testicular size was observed.

All the patients underwent seminal analysis before surgery, and 6 months after surgery (Table 3). In each group, the postoperative sperm count, sperm volume, motility, and the percentage of sperm with abnormal morphology were significantly improved compared with the preoperative test values. The postoperative findings of the 2 groups were similar.

Table 1. Patient characteristics and clinical features.

\begin{tabular}{lccc}
\hline & L-SVL & OI-SVL & P \\
\hline Patients, $n$ & 140 & 120 & - \\
\hline
\end{tabular}




\begin{tabular}{lllll}
\hline Weight, kg & & $62.6 \pm 11.3$ & $64.5 \pm 12.1$ & $0.35^{\mathrm{a}}$ \\
\hline Age, y & & $27.6 \pm 5.8$ & $26.3 \pm 6.2$ & $0.76^{\mathrm{a}}$ \\
\hline Varicocele side & Left & 124 & 99 & $<0.05^{\mathrm{b}}$ \\
\cline { 2 - 5 } & Bilateral & 16 & 21 & \\
\hline Grade (n) & 1 & 19 & 26 & $<0.05^{\mathrm{b}}$ \\
\cline { 2 - 5 } & 2 & 57 & 45 &
\end{tabular}

\begin{tabular}{lllll}
\cline { 2 - 4 } 3 & 64 & 49 & \\
\hline Indications & Abnormal seminal fluid & 140 & 120 & $<0.05^{\mathrm{b}}$ \\
\cline { 2 - 4 } & Infertility & 94 & 87 & \\
\cline { 2 - 4 } & Scrotal pain & 123 & 108 \\
\cline { 2 - 4 } & Testicular hypotrophy & 7 & 5 \\
\hline
\end{tabular}

aStudent's t-test; ${ }^{\mathrm{b}} \mathrm{Chi}$-squared test

Table 2. Intra- and post-operative morbidity in the L-SVL and OI-SVL groups*.

\begin{tabular}{|c|c|c|c|c|}
\hline & & L-SVL & OI-SVL & $\mathbf{P}$ \\
\hline Varicocele recurrence rate & & $0.83(1 / 120)$ & $2.86(4 / 140)$ & 0.001 \\
\hline Operative time, $\min$ & & $41.3 \pm 9.6$ & $54.9 \pm 13.5$ & 0.034 \\
\hline Return to normal activity, $d$ & & $1.8 \pm 0.9$ & $3.2 \pm 1.4$ & 0.028 \\
\hline Hospitalization, d & & $4.3 \pm 0.82$ & $7.5 \pm 2.1$ & 0.015 \\
\hline Cost, RMB, Yuan & & $7856 \pm 345$ & $7262 \pm 312$ & 0.079 \\
\hline \multirow[t]{5}{*}{ Complications } & Wound complications & $5.83(7 / 120)$ & $7.86(11 / 140)$ & 0.001 \\
\hline & Genitofemoral nerve damage & $0(0 / 120)$ & $1.43(2 / 140)$ & 0.289 \\
\hline & Testicular atrophy & $0(0 / 120)$ & $2.14(3 / 140)$ & 0.155 \\
\hline & Epididymo-orchitis & $1.67(2 / 120)$ & $2.14(3 / 140)$ & 0.336 \\
\hline & Hydrocele & $3.33(4 / 120)$ & $1.432 / 140)$ & 0.197 \\
\hline
\end{tabular}

${ }^{*}$ Reported as \% or mean \pm standard error of the mean

Table 3. Preoperative and postoperative semen tests.

\begin{tabular}{|c|c|c|c|c|c|c|c|}
\hline & \multicolumn{3}{|l|}{ L-SVL } & \multicolumn{3}{|l|}{ OI-SVL } & \multirow[t]{2}{*}{$\mathbf{P}^{*}$} \\
\hline & Preop & Postop & $\mathbf{P}$ & Preop & Postop & $\mathbf{P}$ & \\
\hline Volume, ml & $2.1 \pm 0.4$ & $2.9 \pm 0.5$ & $<0.01$ & $2.2 \pm 0.5$ & $2.8 \pm 0.6$ & $<0.01$ & 0.347 \\
\hline Sperm count, $\times 10^{6} / \mathrm{ml}$ & $10.8 \pm 3.6$ & $37.8 \pm 8.6$ & $<0.01$ & $12.2 \pm 4.3$ & $38.1 \pm 9.6$ & $<0.01$ & 0.065 \\
\hline Motility, \% & $23.7 \pm 6.6$ & $45.6 \pm 11.4$ & $<0.01$ & $24.8 \pm 7.3$ & $44.5 \pm 12.6$ & $<0.01$ & 0.964 \\
\hline Normal morphology, \% & $9.8 \pm 2.1$ & $25.6 \pm 4.3$ & $<0.01$ & $8.9 \pm 2.3$ & $26.4 \pm 5.1$ & $<0.01$ & 1.023 \\
\hline
\end{tabular}

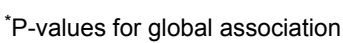

\section{Discussion}

Although left varicocele is very common in clinical practice, the precise link between varicocele and subfertility is not well understood. It has been reported that retrograde venous flow causes dysfunction of the Leydig cells, thus resulting in elevated testicular temperature and exposure to renal and adrenal metabolites [9]. It is worthwhile to treat symptomatic cases of varicocele whether or not there is impairment of seminal fluid. In fact, spermatic vessel ligation is known to improve fertility in as many as $70 \%$ of infertility [10]. In the present study, the postoperative semen analyses confirmed that both L-SVL and OI-SVL were similarly able to improve sperm count, grade of motility, and morphology. The study also showed that minimally invasive procedures could be successfully achieved by laparoscopic surgery.

Surgical vein interruption can be accomplished by inguinal, retroperitoneal, or subinguinal approaches. Ideally, treating varicoceles includes the ligation of all testicular veins, but with the preservation of testicular arteries and the lymphatic system [11]. In recent years, many non-invasive or minimally invasive techniques have been proposed to minimize complications. Since the laparoscopic technique was first described in 1988 [12], it has been the preferred method for pediatric and adolescent patients [5]. The laparoscopic technique allows easier identification of the spermatic artery by magnification of tissues and vessels on a video monitor, in a manner comparable to that of an operating microscope. The advantages of laparoscopic varicocelectomy include clear and increased 
magnification of the surgical field. Identification of vessels is more accurate, such as the testicular collateral veins which run alongside the spermatic cord and together enter the internal ring lymphatics, and the testicular artery. The present study confirmed that the use of laparoscopy to clip and divide the internal spermatic vessel is simple, safe, and effective.

It has been reported that high retroperitoneal ligation of the internal spermatic vessels (i.e., the Palomo technique) [13] results in a significant reduction in the surgical failure rate for varicoceles, compared with artery-sparing procedures [14,15]. However, in theory it is preferable to preserve the testicular artery, even though the testis is vascularized by two other arteries. In fact, vascular communication among the testicular, cremasteric, and vasal arteries has been reported [16]. Even patent small veins left around the artery may result in the recurrence of varicocele [17]. However, Matsuda et al.'s study [18] did not show any significant difference between arterypreserving varicocelectomy and the artery-ligating procedure, with both techniques showing improvements in semen quality and postoperative pregnancy rates. The incidence of recurrent varicocele can be as high as $8 \%$ to $28 \%$ after artery-preserving open varicocelectomy [15]. In the current study, the recurrence rate of the L-SVL group $(0.83 \%)$ was significantly lower than that of the OI-SVL group $(\mathrm{P}<0.01)$, and much lower than that reported by Matsuda et al. [18].

In our study, the preferred site for vein ligation was $3 \mathrm{~cm}$ superior to the inner inguinal ring. The dilated refluxing spermatic vessel has a number of venous connections proximal to the inner inguinal ring; however, distally, very few connections are present with extra-funicular veins. Therefore, the internal inguinal ring is a gateway whereby the refluxing spermatic vessels can be totally excluded from the testicular venous drainage. In fact, the recurrence rate is known to be lower after using the inguinal approach than after the retroperitoneal approach [19]. Furthermore, laparoscopic vein ligation should be implemented at the internal inguinal ring, where the spermatic vessels are visible from the peritoneal cavity. We observed no testicular atrophy after either the LSVL or OI-SVL procedure in the present study.

This study has several limitations which should be considered when interpreting our results. Firstly, this was a retrospective study, and is therefore generally less reliable than prospective studies. Secondly, the study was also conducted at a single institution and a single occupational cluster, which may have caused selection bias. Thirdly, our hospital is without an appropriate microscope at present, thus MSV is not currently carried out in our department. Accordingly, future studies should have a larger sample size and a prospective design; this will permit further investigation of the relative benefits of different types of surgery for infertile men.

\section{Conclusion}

The results of this relatively small-scale study suggest that LSVL is an ideal procedure for the repair of varicocele and the restoration of fertility; it is a simple, safe, effective, and time- sparing procedure. The distinct advantages of L-SVL relative to OI-SVL include rapid recovery, a lower complication and recurrence rate, and better improvement in the semen prolife. There is little likelihood of the condition recurring, and no morbidity or mortality was encountered in our study. However, the important feature of L-SVL surgery is the complete and thorough bilateral high ligation of the testicular veins, without any risk of injury to the testicular artery, and this requires familiarity and skills which need time and experience to acquire.

\section{Acknowledgments}

This work was funded by the Outstanding Leaders Training Program of Pudong Health Bureau of Shanghai (No. PWR120616-05), and supported by the grant from the Key Discipline Construction Project of Shanghai Health Bureau (No. ZK2015A11).

\section{Conflict of Interest}

The authors declare that there is no conflict of interest.

\section{References}

1. Baazeem A, Belzile E, Ciampi A, Dohle G, Jarvi K, Salonia A. Varicocele and male factor infertility treatment: a new meta-analysis and review of the role of varicocele repair. Eur Urol 2011; 60: 796-808.

2. Hopps CV, Lemer ML, Schlegel PN, Goldstein M. Intraoperative varicocele anatomy: a microscopic study of the inguinal versus subinguinal approach. J Urol 2003; 170: 2366-2370.

3. Pfeiffer D, Berger J, Schoop C, Tauber R. A Doppler-based study on the prevalence of varicocele in German children and adolescents. Andrologia 2006; 38: 13-19.

4. Lv JX, Wang LL, Wei XD. Comparison of treatment outcomes of different spermatic vein ligation procedures in varicocele treatment. Am J Ther 2016; 23: 1329-1334.

5. Borruto FA, Impellizzeri $P$, Antonuccio $P$, Finocchiaro A, Scalfari G, Arena F. Laparoscopic vs open varicocelectomy in children and adolescents: review of the recent literature and meta-analysis. J Pediatr Surg 2010; 45: 2464-2649.

6. Mendez-Gallart R, Bautista-Casasnovas A, EstevezMartinez E, Varela-Cives R. Laparoscopic Palomo varicocele surgery: lessons learned after 10 years follow up of 156 consecutive pediatric patients. J Pediatr Urol 2009; 5: $126-131$.

7. Grasso M, Lania C, Castelli M, Galli L, Franzoso F, Rigatti P. Low-grade left varicocele in patients over 30 years old: the effect of spermatic vein ligation on fertility. BJU Int 2000; 85: 305-307.

8. Hinman FJ. Atlas of Urosurgical anatomy (3rd edn.). Philadelphia, PA: WB Saunders 1993.

9. Pryor JL, Howards SS. Contemporary Management of Impotence and Infertility. Baltimore: Williams Wilkins 1988. 
10. Sigman M, Howards SS. Male infertility. Campbells urology. Philadelphia: WB Saunders 1992.

11. Cayan S, Shavakhabov S, Kadioglu A. Treatment of palpable varicocele in infertile men: a meta-analysis to define the best technique. J Androl 2009; 30: 33-40.

12. Sanchez de Badajoz E, Diaz Ramirez F, Marin Marin J. Endoscopic treatment of varicocele. Archivos Espanoles de Urologia 1988; 41: 15-16.

13. Palomo A. Radical cure of varicocele by a new technique; preliminary report. J Urol 1949; 61: 604-607.

14. Hosli PO. Follow-up studies after operation of varicocele in adolescence. Dialogue Pediatr Urol 1990; 13: 4.

15. Kass EJ, Marcol B. Results of varicocele surgery in adolescents: a comparison of techniques. J Urol 1992; 148: 694-696.

16. Lee LM, Johnson HW, McLonghlin MG. Microdissection and radiographic studies of the arterial vasculature of the human testes. J Pediatr Surg 1984; 19: 297-302.
17. Belloli G, DAgostino S, Musi L, Campobasso P. Adolescent varicocele: operative anatomy and tricks for successful correction. Eur Off J Aus Assoc Pediatr Surg 1995; 5: 219-221.

18. Matsuda T, Horii Y, Yoshida. Should the testicular artery be preserved at varicocelectomy? J Urol 1993; 149: 1357-1361.

19. Sayfan JA, Soffer Y. A natural venous bypass causing postoperative recurrence of a varicocele. J Androl 1981; 2: 108.

\section{*Correspondence to}

Chuan-Yi Hu

Department of Urology

Gongli Hospital of Shanghai Pudong

PR China 